\title{
Robust Observer Based Disturbance Rejection Control for Euler-Lagrange Systems
}

\author{
Yanjun Zhang, Lu Wang, Jun Zhang, and Jianbo Su \\ Department of Automation, Shanghai Jiao Tong University, Shanghai, China \\ Correspondence should be addressed to Jianbo Su; jbsu@sjtu.edu.cn
}

Received 24 January 2016; Revised 11 May 2016; Accepted 5 June 2016

Academic Editor: Sergey A. Suslov

Copyright ( 2016 Yanjun Zhang et al. This is an open access article distributed under the Creative Commons Attribution License, which permits unrestricted use, distribution, and reproduction in any medium, provided the original work is properly cited.

\begin{abstract}
Robust disturbance rejection control methodology is proposed for Euler-Lagrange systems, and parameters optimization strategy for the observer is explored. First, the observer based disturbance rejection methodology is analyzed, based on which the disturbance rejection paradigm is proposed. Thus, a disturbance observer (DOB) with partial feedback linearization and a lowpass filter is proposed for nonlinear dynamic model under relaxed restrictions of the generalized disturbance. Then, the outer-loop backstepping controller is designed for desired tracking performance. Considering that the parameters of DOB cannot be obtained directly based on Lyapunov stability analysis, parameter of DOB is optimized under standard $H_{\infty}$ control framework. By analyzing the influence of outer-loop controller on the inner-loop observer parameter, robust stability constraint is proposed to guarantee the robust stability of the closed-loop system. Experiment on attitude tracking of an aircraft is carried out to show the effectiveness of the proposed control strategy.
\end{abstract}

\section{Introduction}

Euler-Lagrange systems widely exist in practice, such as manipulator, mobile robot, underwater vehicle, surface vessel, and aerial vehicle. Consequently, motion control of EulerLagrange systems has been widely explored in the past decades. Motion control systems usually work at unknown environment, and inevitably, they suffer from system uncertainties and external disturbances, which will affect the control performance or even make the system unstable [1]. To deal with this problem, numerous approaches have been proposed, such as sliding mode control [2-4], adaptive control [5-7], robust control [8-10], and intelligent control [11-13]. These control methods can more or less deal with the system uncertainties. However, facing the problems is still inevitable, such as chattering of sliding mode control, stability problem of adaptive control, conservative robust control, and convergence rate of weights in neural network and fuzzy system.

The effectiveness of disturbance observer (DOB) has been shown in many applications, such as humanoid robot control $[14,15]$, manipulator control [16-18], aircraft control $[19,20]$, optical disk control [21, 22], motor control [23, 24], and vibration control $[25,26]$. Traditional DOB methodology, which is proposed based on linear system, cannot be used directly in nonlinear systems [27]. In [28], traditional linear DOB is applied for disturbance rejection of nonlinear system. However, only first-order binomial coefficient typed lowpass filter is used for DOB implementation. The performance of the closed-loop system cannot be improved effectively. Meanwhile, the optimization strategy of parameters is not investigated. Nonlinear DOB is proposed in $[29,30]$, which can be directly used for disturbance estimation in nonlinear systems. In this paper, we find that estimation effect of nonlinear $\mathrm{DOB}$ is the same as that of linear DOB with first-order low-pass filter when a constant observer gain is selected. And asymptotic stability is guaranteed simply based on the assumption that the generalized disturbances and their first-order derivatives are bounded and that the first derivatives go to zero in the steady state, which is not realistic in most conditions. Meanwhile, for a closed-loop system, the parameters of inner-loop observer depend on not only system uncertainties and measurement noise, but also the structure and parameter of outer-loop controller. However, the existing works rarely discuss parameters optimization of 
the observer. The influence caused by outer-loop controller is never explored in existing researches.

From the descriptions above, a robust DOB based disturbance rejection controller is proposed, and parameters optimization strategy is investigated. Nonlinear DOB and extended state observer (ESO) are first analyzed to show the essence of the disturbance estimation problem. Then, under relaxed restrictions of disturbance and system perturbation, a novel disturbance observer is proposed for nonlinear system. The observer consists of a feedback linearization compensator and a low-pass filter. The feedback linearization compensator is introduced to linearize the nonlinear dynamics into a linear part disturbed by the equivalent disturbance, whereas the low-pass filter is employed to estimate the equivalent disturbances. Then, a state feedback controller is presented for the nominal model to acquire desired performance. Stability of the overall closed-loop system is analyzed based on Lyapunov theory. At last, the influence on DOB parameters optimization caused by structure and parameter of outer-loop controller is analyzed. The robust stability constraint condition, which ensures the robust stability of the whole system, is proposed. Thus, the $H_{\infty}$ method can be employed to optimize the parameters of the DOB.

The main contributions of this paper are summarized as follows:

(1) The disturbance rejection paradigm of the observer based disturbance rejection methodology is proposed.

(2) With the proposed disturbance rejection paradigm, a novel observer, whose low-pass filter of its structure can be selected to be flexible, is proposed for nonlinear systems.

(3) The parameters optimization method is investigated to make sure the designed control system can guarantee the robust stability of the closed-loop system.

The rest of this paper is organized as follows. In Section 2, a mechanical system model is established, based on which the disturbance rejection problem is formulated. In Section 3, DOB based control methodology is proposed, and parameters of $\mathrm{DOB}$ are optimized to guarantee the robust stability. In Section 4, attitude tracking task is carried out to show the effectiveness of the proposed strategy, followed by conclusions in Section 5.

\section{System Model and Problem Statement}

2.1. System Model. An Euler-Lagrange equation for the mechanical system is described as

$$
M(q) \ddot{q}+C(q, \dot{q}) \dot{q}+G(q)=u+d,
$$

where $q \in \mathbb{R}^{n}$ and $\dot{q} \in \mathbb{R}^{n}$ denote the generalized coordinates and velocities and $u$ and $d$ are the control input and external disturbance, respectively. $M(q) \in \mathbb{R}^{n \times n}$ represent the positive definite inertial matrix, $C(q, \dot{q}) \dot{q} \in \mathbb{R}^{n \times 1}$ represents the matrix of Coriolis and centrifugal forces, and $G(q) \in \mathbb{R}^{n \times 1}$ represents the gravity term. The nonlinear functions $M(\cdot), C(\cdot)$, and $G(\cdot)$ satisfy the following assumption.
Assumption 1. The unknown nonlinear functions $M(\cdot), C(\cdot)$, and $G(\cdot)$ are continuously differentiable and locally Lipschitz.

By introducing the definitions

$$
\begin{aligned}
& x_{1}=q, \\
& x_{2}=\dot{q},
\end{aligned}
$$

(1) can be rewritten as

$$
\begin{aligned}
\dot{x}_{1}= & x_{2}, \\
\dot{x}_{2}= & -M^{-1}\left(x_{1}\right)\left(C\left(x_{1}, x_{2}\right) x_{2}+G\left(x_{1}\right)\right) \\
& +M^{-1}\left(x_{1}\right)(u+d) .
\end{aligned}
$$

According to the parameters perturbation, it is impossible to establish the system model accurately. By introducing the notations

$$
\begin{aligned}
M(q) & =M_{0}(q)+M_{\Delta}(q), \\
C(q, \dot{q}) & =C_{0}(q, \dot{q})+C_{\Delta}(q, \dot{q}), \\
G(q) & =G_{0}(q)+G_{\Delta}(q),
\end{aligned}
$$

where subscript 0 denotes the nominal value of the corresponding matrix and subscript $\Delta$ denotes the part of perturbation, then, the dynamics can be described as follows:

$$
\begin{aligned}
& \dot{x}_{1}=x_{2}, \\
& \dot{x}_{2}=F(x)+G(x) u+f+d^{\prime},
\end{aligned}
$$

where $F(x)=-M_{0}^{-1}\left(x_{1}\right)\left(C_{0}\left(x_{1}, x_{2}\right) x_{2}+G_{0}\left(x_{1}\right)\right), G(x)=$ $M_{0}^{-1}\left(x_{1}\right)$, and $d^{\prime}=M^{-1}\left(x_{1}\right) d$. $f$ is the perturbed term caused by the internal uncertainty, which is defined as

$$
\begin{aligned}
f & =M^{-1}\left(x_{1}\right) \\
& \cdot\left[M_{0}^{-1}\left(x_{1}\right) M_{\Delta}\left(x_{1}\right)\left(C_{0}\left(x_{1}, x_{2}\right) x_{2}+G_{0}\left(x_{1}\right)+u\right)\right. \\
& \left.-C_{\Delta}\left(x_{1}, x_{2}\right) x_{2}-G_{\Delta}\left(x_{1}\right)\right] .
\end{aligned}
$$

In practical applications, the consumption of the external disturbances is finite; that is, the external disturbance $d$ is bounded. Nevertheless, internal uncertainty $f$ usually depends on system state. Assume that the controller $u$ is defined as $u=v\left(x_{1}, x_{2}, \widehat{d}\right)$; nonlinear function $v(\cdot)$ is continuously differentiable. Thus, from the definition of $f$, we can also obtain that $f$ is continuously differentiable. From the above analysis, the following assumptions can be obtained.

Assumption 2. The external disturbance $d^{\prime}=d_{1}+d_{2}(t)$ is bounded, where $d_{1}$ and $d_{2}(t)$ represent the constant and timevarying component. The time-varying component satisfies $\left\|d_{2}(t)\right\| \leq \bar{d}$.

Assumption 3. The internal uncertainties $f$ satisfy $\|f\| \leq$ $\alpha\left(x_{1}, x_{2}, \bar{d}\right)$, where $\alpha(\cdot)$ is classical $\mathscr{K}$ function. 
2.2. Problem Formulation. For the system model described in (5), the key point of the antidisturbance control methodology is the observer configuration. The control accuracy and robustness of the overall system are largely determined by the performance of observer. Here, several widely used observers are provided for analysis. Based on the disturbance rejection paradigm, we propose a novel observer structure and parameter optimization strategy for nonlinear systems.

2.2.1. Extended State Observer (ESO). ESO is the most important part of the active disturbance rejection control (ADRC) [31]. Instead of identifying the plant dynamics off-line, ESO can estimate the combined effect of plant dynamics and external disturbance in real time. However, ESO can be only used for the standard chained systems. Here, an ESO is designed as

$$
\begin{aligned}
& \dot{\vec{z}}_{1}=F(x)+G(x) u+\widehat{z}_{2}+g_{1}\left(x_{1}-\widehat{z}_{1}\right), \\
& \dot{\vec{z}}_{2}=g_{2}\left(x_{1}-\widehat{z}_{1}\right),
\end{aligned}
$$

where $g_{1}$ and $g_{2}$ are positive constant to be selected such that $s^{2}+g_{1} s+g_{2}$ is Hurwitz.

By substituting (5) into (7) and introducing the Laplace Transformation, we finally get the following equation:

$$
\widehat{z}_{2}=\frac{g_{2}}{s^{2}+g_{1} s+g_{2}}\left(f+d^{\prime}\right)
$$

where $s$ is the Laplace operator.

2.2.2. Nonlinear Disturbance Observer (NDOB). The NDOB has been widely used for nonlinear systems with uncertainties [30]. It can estimate the composite disturbances and compensate in the feedback controller. The NDOB for the dynamics of (5) is given as

$$
\begin{aligned}
& \widehat{d}=z+p(x), \\
& \dot{z}=-L(x)(z+p(x))+L(x)(-F(x)-G(x) u),
\end{aligned}
$$

where $L(x) \triangleq \partial p(x) / \partial x$.

From (9) we get

$$
\dot{\hat{d}}=-L(x) \widehat{d}+L(x)\left(f+d^{\prime}\right) .
$$

Then, by introducing the Laplace Transformation, we finally get

$$
\widehat{d}=\frac{L(x)}{s+L(x)}\left(f+d^{\prime}\right) .
$$

In most applications, observer gain $L(x)$ is usually selected as a positive constant.

2.2.3. Disturbance Rejection Paradigm. According to the analysis above, we find that the estimation of the observer can be obtained as the real composite disturbance passing through a low-pass filter. It can be summarized that the estimation effect of the observers should fulfill the following disturbance rejection paradigm:

$$
\widehat{d}=Q(s) D
$$

where $D \triangleq f+d^{\prime}$ is the composite disturbance which contains both external disturbances and equivalent internal disturbances. $Q(s)$ is a low-pass filter such that $\widehat{d}$ can converge to $D$ asymptotically.

For most researches on observer based control, the structure of the low-pass filter $Q(s)$ is usually fixed by the observer structure. Meanwhile, the parameters tuning usually relies on trial and error; rarely do researches focus on the point of how to optimize the observer parameters according to the property of system uncertainties, outer-loop controller, measurement noise, and so forth. Hence, in this paper, a novel observer, whose low-pass filter can be selected to be flexible, is proposed for the nonlinear system. Particularly, the parameters optimization strategy is explored for nonlinear systems.

\section{Controller Design and Parameter Optimization}

3.1. Controller Design. The objective of controller design is that the observer is proposed to estimate the internal uncertainty $f$ and external disturbance $d^{\prime}$, and thus the estimation $\widehat{d}$ is compensated in the closed-loop control system. Then, feedback controller $u$ is designed to stabilize the system to the equilibrium point $\left(x_{1}=0, x_{2}=0\right)$. The control structure is shown in Figure 1.

The inner-loop observer is designed firstly. By introducing a feedback linearization

$$
u=G^{-1}(x)(v-F(x)),
$$

the nonlinear system can be compensated as

$$
\begin{aligned}
& \dot{x}_{1}=x_{2}, \\
& \dot{x}_{2}=v+D(x, t),
\end{aligned}
$$

where $D(x, t)=f+d^{\prime}$ is the composite disturbance.

Then, the observer is designed as

$$
\widehat{d}=-Q(s) v+s Q(s) x_{2},
$$

where $Q(s)$ is a low-pass filter to be optimized.

According to (14) and (15), it can be obtained that $\widehat{d}=$ $-Q(s) v+Q(s) \dot{x}_{2}=Q(s) D(s)$; that is, the observer satisfies the disturbance rejection paradigm in (12). In practical applications, $Q(s)$ and $s Q(s)$ can be realized in state-space.

Then, the backstepping controller can be designed for the nominal system. Introduce the following notations:

$$
\begin{aligned}
& e_{1}=x_{1}-x_{1 \mathrm{~d}}, \\
& e_{2}=x_{2}-\beta_{1},
\end{aligned}
$$

where $\beta_{1}$ is the pseudo controller to be designed, $x_{1 \mathrm{~d}}$ is a differentiable reference input. 


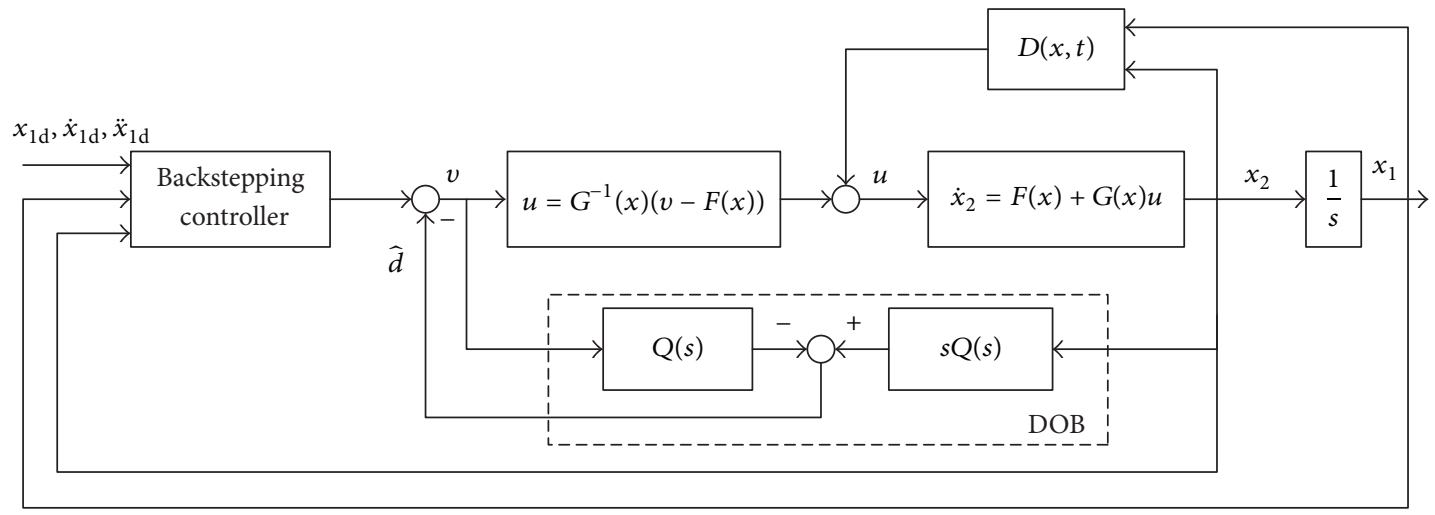

FIGURE 1: Control structure of the closed-loop system.

From the definition of $e_{1}$ and $e_{2}$, derivative of $e_{2}$ is described as

$$
\dot{e}_{1}=x_{2}-\dot{x}_{1 \mathrm{~d}}=e_{2}+\beta_{1}-\dot{x}_{1 \mathrm{~d}} \text {. }
$$

The pseudo controller $\beta_{1}$ is hence defined as

$$
\beta_{1}=-K_{1} e_{1}+\dot{x}_{1 \mathrm{~d}}
$$

where $K_{1}$ is a positive symmetric matrix.

Substituting (18) into (17) yields

$$
\dot{e}_{1}=-K_{1} e_{1}+e_{2} .
$$

Define a Lyapunov function $V_{1}=(1 / 2) e_{1}^{\mathrm{T}} e_{1}$; its derivative is $\dot{V}_{1}=-e_{1}^{\mathrm{T}} K_{1} e_{1}+e_{1} e_{2}$. Notice that the derivative of $e_{2}$ is

$$
\dot{e}_{2}=\dot{x}_{2}-\dot{\beta}_{1}=v+D(x, t)-\dot{\beta}_{1},
$$

where $\dot{\beta}_{1}=-K_{1} e_{2}+K_{1}^{2} e_{1}+\ddot{x}_{1 \mathrm{~d}}$. According to the backstepping approach and observer output $\widehat{d}$, the controller is finally obtained as

$$
u=G^{-1}(x)\left(-K_{2} e_{2}-e_{1}+\dot{\beta}_{1}-\widehat{d}-F(x)\right) .
$$

For the Lyapunov function $V_{2}=(1 / 2) e_{1}^{\mathrm{T}} e_{1}+(1 / 2) e_{2}^{\mathrm{T}} e_{2}$, its time-derivative satisfies

$$
\dot{V}_{2} \leq-e_{1}^{\mathrm{T}} K_{1} e_{1}-e_{2}^{\mathrm{T}} K_{2} e_{2}+\left\|e_{2}\right\|\|\tilde{d}\|,
$$

where $\tilde{d} \triangleq D(x, t)-\widehat{d}$ is disturbance estimating error of the observer. Assume that the estimating error of observer is the input of the above system; then the unforced system is exponentially stable at the equilibrium point.

\subsection{Stability Analysis}

Theorem 4. For the given second-order mechanical system in (5), the external disturbances and equivalent internal uncertainties satisfy Assumptions 2 and 3. By adopting the observer in (15) and controller in (21), the control error of system states and estimation error of observer are locally uniformly ultimately bounded (UUB).
Proof. For the outer-loop controller, by substituting $e_{1}$ and $e_{2}$ into (21), it can be obtained that

$$
\begin{aligned}
v= & -\left(1+K_{1} K_{2}\right) x_{1}-\left(K_{1}+K_{2}\right) x_{2} \\
& +\left(1+K_{1} K_{2}\right) x_{1 \mathrm{~d}}+\left(K_{1}+K_{2}\right) \dot{x}_{1 \mathrm{~d}}+\ddot{x}_{1 \mathrm{~d}}-\widehat{d} .
\end{aligned}
$$

Then, the dynamics can be rewritten as

$$
\begin{aligned}
\dot{x}_{2}= & -\left(1+K_{1} K_{2}\right) x_{1}-\left(K_{1}+K_{2}\right) x_{2} \\
& +\left(1+K_{1} K_{2}\right) x_{1 \mathrm{~d}}+\left(K_{1}+K_{2}\right) \dot{x}_{1 \mathrm{~d}}+\ddot{x}_{1 \mathrm{~d}} \\
& +D(x, t)-\hat{d} .
\end{aligned}
$$

For the system state defined as $x=\left[\begin{array}{ll}x_{1} & x_{2}\end{array}\right]^{\mathrm{T}}$, the following differential equation can be obtained:

$$
\begin{aligned}
\dot{x}= & A_{1} x+B_{1}\left[\left(1+K_{1} K_{2}\right) x_{1 \mathrm{~d}}+\left(1+K_{1}+K_{2}\right) \dot{x}_{1 \mathrm{~d}}\right. \\
& \left.+d_{1}+d_{2}(t)+f-\hat{d}\right],
\end{aligned}
$$

where

$$
\begin{aligned}
& A_{1}=\left[\begin{array}{cc}
0 & 1 \\
-\left(1+K_{1} K_{2}\right) & -\left(K_{1}+K_{2}\right)
\end{array}\right], \\
& B_{1}=\left[\begin{array}{l}
0 \\
1
\end{array}\right] .
\end{aligned}
$$

For the inner-loop observer, the state-space equation is established as

$$
\begin{aligned}
& \dot{z}=A_{2} z+B_{2} D(x, t), \\
& \widehat{d}=C_{2} z,
\end{aligned}
$$

where $z$ is the system state and $\left(A_{2}, B_{2}, C_{2}\right)$ and $z$ depend on the structure of low-pass filter $Q(s) .\left(A_{2}, B_{2}, C_{2}\right)$ is minimum implementation, $\left(A_{2}, B_{2}\right)$ is controllable, and $\left(A_{2}, C_{2}\right)$ is observable. Since $Q(s) \in R H_{\infty}, A_{2}$ is a Hurwitz matrix. 
For the overall closed-loop system, define the generalized state $\xi=\left[\begin{array}{ll}x^{\mathrm{T}} & z^{\mathrm{T}}\end{array}\right]^{\mathrm{T}}$; according to (25) and (27), the state-space equation can be obtained in

$$
\begin{aligned}
\dot{\xi} & =\underbrace{\left[\begin{array}{cc}
A_{1} & -B_{1} C_{2} \\
0 & A_{2}
\end{array}\right]}_{A} \xi+\underbrace{\left[\begin{array}{c}
B_{1} \\
B_{2}
\end{array}\right]}_{B}\left(f(y)+d_{2}(t)\right) \\
& +\underbrace{\left[\begin{array}{c}
B_{1} d_{1}+B_{1}\left(1+K_{1} K_{2}\right) x_{1 \mathrm{~d}}+B_{1}\left(K_{1}+K_{2}\right) \dot{x}_{1 \mathrm{~d}}+B_{1} \ddot{x}_{1 \mathrm{~d}} \\
B_{2} d_{1}
\end{array}\right]}_{r} \\
y & =\underbrace{\left[\begin{array}{cc}
I_{2 \times 2} & 0 \\
0 & C_{2}
\end{array}\right]}_{C} .
\end{aligned}
$$

Since $A_{1}$ and $A_{2}$ are both Hurwitz matrices, we can easily know that $A$ is Hurwitz according to its definition. That is, for any given positive definite symmetric matrix $N$, there exists a positive definite symmetric matrix $P$ such that $P A+A^{\mathrm{T}} P=$ $-N$. The equilibrium point is

$$
\xi_{0}=-A^{-1} B d_{1}+\left[\begin{array}{ll}
1 & 0 \\
0 & 1 \\
0 & 0
\end{array}\right]\left[\begin{array}{c}
x_{1 \mathrm{~d}} \\
\dot{x}_{1 \mathrm{~d}}
\end{array}\right]
$$

For $\widetilde{\xi}=\xi-\xi_{0}$, we have the following state equation:

$$
\begin{aligned}
& \dot{\tilde{\xi}}=A \widetilde{\xi}+B\left(f(y)+d_{2}(t)\right), \\
& y=C\left(\widetilde{\xi}+\xi_{0}\right) .
\end{aligned}
$$

For the nonlinear function $f$, there exists a compact set $\Omega$ such that

$$
\begin{aligned}
\|f(y)\| & \leq \gamma\|y\|, \\
\gamma & =\sup _{y \in \Omega}\left|\frac{\partial f(y)}{\partial y}\right| .
\end{aligned}
$$

For the Lyapunov function defined as $V=\widetilde{\xi}^{\mathrm{T}} P \widetilde{\xi}$, its timederivative satisfies

$$
\begin{aligned}
\dot{V} & =-\widetilde{\xi}^{\mathrm{T}} N \widetilde{\xi}+2 \widetilde{\xi}^{\mathrm{T}} P B\left(f(y)+d_{2}(t)\right) \leq-\left[\lambda_{\min }(N)\right. \\
& -2 \gamma\|P B\|\|C\|]\|\tilde{\xi}\|^{2}+2\|P B\|\left[\left\|C \widetilde{\xi}_{0}\right\|+\left\|d_{2}(t)\right\|\right]\|\tilde{\xi}\| \\
& \leq-\left[\lambda_{\min }(N)-2 \gamma\|P B\|\|C\|\right. \\
& \left.-\frac{2\|P B\|\left(\left\|C \tilde{\xi}_{0}\right\|+\bar{d}\right)}{\|\tilde{\xi}\|}\right]\|\tilde{\xi}\|^{2} .
\end{aligned}
$$

Consequently, the control error of system states and estimation error of observer are locally UUB.
3.3. Parameters Optimization. Theorem 4 provides us with the parameter range such that the closed-loop system is UUB. However, it is very hard to determine the parameters directly. In this section, a parameter optimization strategy of the lowpass filter guaranteeing the robust stability is proposed.

The parameter of the low-pass filter $Q(s)$ is influenced by system uncertainties, parameters of the outer-loop controller, and measurement noise. First, the observer is transformed as

$$
\begin{aligned}
\widehat{d}= & -Q(s) v+s Q(s) x_{2}=-Q(s) \\
& \cdot\left[\left(1+K_{1} K_{2}\right) x_{1 \mathrm{~d}}+\left(K_{1}+K_{2}\right) \dot{x}_{1 \mathrm{~d}}+\ddot{x}_{1 \mathrm{~d}}-\widehat{d}\right] \\
& +Q(s)\left[s+\left(K_{1}+K_{2}\right)+\frac{\left(1+K_{1} K_{2}\right)}{s}\right] x_{2} .
\end{aligned}
$$

Then, (24) can be transformed as the following equivalent structure:

$$
\begin{aligned}
{[s+} & \left.\left(K_{1}+K_{2}\right)+\frac{\left(1+K_{1} K_{2}\right)}{s}\right] x_{2} \\
= & \left(1+K_{1} K_{2}\right) x_{1 \mathrm{~d}}+\left(K_{1}+K_{2}\right) \dot{x}_{1 \mathrm{~d}}+\ddot{x}_{1 \mathrm{~d}} \\
& +D(x, t)-\widehat{d} .
\end{aligned}
$$

The nominal model of equivalent system is

$$
P_{\Delta n}(s)=\frac{s}{s^{2}+\left(K_{1}+K_{2}\right) s+\left(1+K_{1} K_{2}\right)} .
$$

Then, we mainly analyze the system uncertainty of the equivalent system. The system uncertainty is defined as

$$
\begin{aligned}
f\left(x_{1}, x_{2}\right)= & -s M_{\Delta}\left(x_{1}\right) x_{2}+C_{\Delta}\left(x_{1}, x_{2}\right) x_{2} \\
& +G_{\Delta}\left(x_{1}\right) .
\end{aligned}
$$

By assuming that the system works in a compact set $\Omega_{x}$, the uncertainty can be linearized as

$$
\begin{aligned}
& f\left(x_{1}, x_{2}\right)=\left[-s M_{\Delta}\left(x_{1}\right)+C_{\Delta}\left(x_{1}, x_{2}\right)\right. \\
& \left.+x_{2} \frac{\partial C_{\Delta}\left(x_{1}, x_{2}\right)}{\partial x_{2}}\right] x_{2}+\left[-s x_{2} \frac{\partial M_{\Delta}\left(x_{1}\right)}{\partial x_{1}}\right. \\
& \left.+x_{2} \frac{\partial C_{\Delta}\left(x_{1}, x_{2}\right)}{\partial x_{1}}+\frac{\partial G_{\Delta}\left(x_{1}\right)}{\partial x_{1}}\right] x_{1} .
\end{aligned}
$$

Since $s x_{1}=x_{2}$, the internal uncertainty satisfies the following linear form:

$$
f\left(x_{1}, x_{2}\right)=-\left(K_{3} s+K_{4}+\frac{K_{4}}{s}\right) x_{2},
$$

where

$$
\begin{aligned}
& K_{3}=M_{\Delta}\left(x_{1}\right) \\
& K_{4}=x_{2} \frac{\partial M_{\Delta}\left(x_{1}\right)}{\partial x_{1}}-C_{\Delta}\left(x_{1}, x_{2}\right)-x_{2} \frac{\partial C_{\Delta}\left(x_{1}, x_{2}\right)}{\partial x_{2}} \\
& K_{5}=-x_{2} \frac{\partial C_{\Delta}\left(x_{1}, x_{2}\right)}{\partial x_{1}}-\frac{\partial G_{\Delta}\left(x_{1}\right)}{\partial x_{1}} .
\end{aligned}
$$



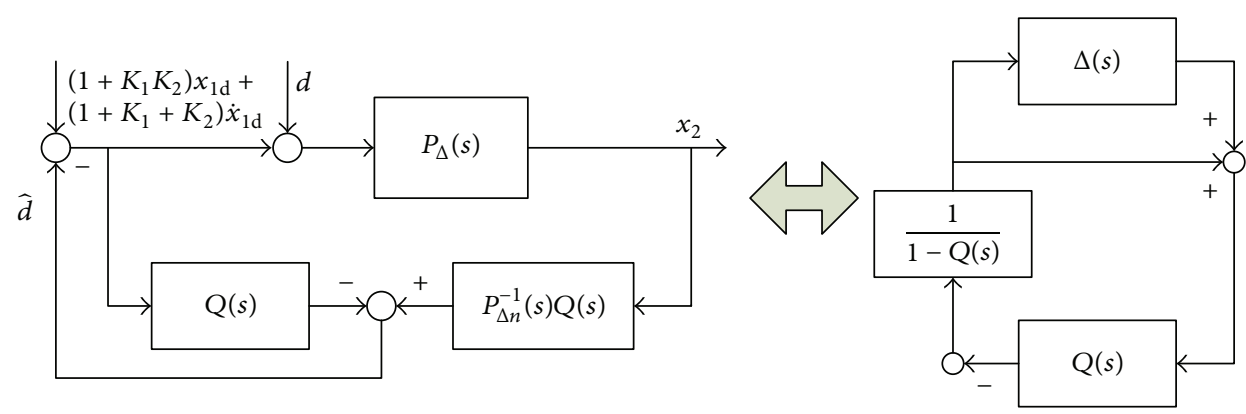

FIGURE 2: Equivalent system transformation.

It is clear that the real plant $P_{\Delta}(s)$ differs if different $x_{1}$ and $x_{2}$ are selected. Define the set of equivalent systems as

$$
\begin{aligned}
& P_{\Delta}(s) \\
& \quad \in\left\{\frac{s}{\left(K_{3}+1\right) s^{2}+\left(K_{1}+K_{2}+K_{4}\right) s+\left(1+K_{1} K_{2}+K_{5}\right)},\right. \\
& \left.\quad\left(x_{1}, x_{2}\right) \in \Omega_{x}\right\} .
\end{aligned}
$$

At this time, the equivalent system can be represented as the form in Figure 2. For the set of equivalent systems and the nominal plant, define the upper bound of the system uncertainty as

$$
\Delta(j \omega) \geq \frac{P_{\Delta}(j \omega)-P_{\Delta n}(j \omega)}{P_{\Delta n}(j \omega)}, \quad \forall \omega,
$$

where scalar $\omega$ denote frequency. From small gain theory, the sufficient condition of robust stability is

$$
\|Q(s) \Delta(s)\|_{\infty}<1 .
$$

Then, the optimization problem can be given as

$$
\begin{array}{ll}
\max & \gamma, \\
\text { s.t. } & \min _{Q(s)}\left\|\left[\begin{array}{c}
\gamma W_{1}(s) \cdot(1-Q(s)) \\
W_{2}(s) \cdot Q(s)
\end{array}\right]\right\|_{\infty}<1 \text {, }
\end{array}
$$

where $W_{1}(s)$ is a stable weighting function that reflects frequency spectrum of disturbances at low frequencies. Weighting function $W_{2}(s)$ satisfies $W_{2}(j \omega)<\Delta(j \omega), \forall \omega$. It can be noticed that the selection of $W_{2}(s)$ is influenced by system uncertainties and outer-loop controller taken into account; meanwhile, the measurement noise should also be taken into account.

By defining the transfer function of virtual loop as $\widetilde{L}(s)=$ $Q(s) /(1-Q(s))=\widetilde{P}(s) \widetilde{K}(s)$, the $Q$ filter design problem turns to be a standard $H_{\infty}$ problem

$$
\begin{array}{ll}
\max & \gamma, \\
\text { s.t. } & \min _{(s) \in R H_{\infty}}\left\|\left[\begin{array}{c}
\gamma W_{1}(s)(1+\widetilde{L}(s))^{-1} \\
W_{2}(s) \widetilde{L}(s)(1+\widetilde{L}(s))^{-1}
\end{array}\right]\right\|_{\infty}<1,
\end{array}
$$

where $\widetilde{L}(s)=\widetilde{P}(s) \widetilde{K}(s)$ and $\widetilde{P}(s)$ and $\widetilde{K}(s)$ are the virtual controlled objective and controller, respectively. The standard state-space solution in $H_{\infty}$ control can be applied to get the optimal solution [32]. For a given virtual controlled objective $\widetilde{P}(s)$, if we can acquire the optimal solution of the virtual controller $\widetilde{K}(s)$, then the optimal $Q$ filter can be obtained as

$$
Q(s)=\frac{\widetilde{P}(s) \widetilde{K}(s)}{1+\widetilde{P}(s) \widetilde{K}(s)} .
$$

Remark 5. If the weighting function $W_{1}(s)$ contains poles on the imaginary axis, the augmented controlled objective of equivalent $H_{\infty}$ control problem will correspondingly contain uncontrollable zeros on the imaginary axis. There is no optimal solution for this $H_{\infty}$ control problem. Thus, the weighting function $W_{2}(s)$ should be transformed as follows:

(1) For the poles at 0

$$
\frac{1}{s} \Longrightarrow \frac{1}{s+\varepsilon}
$$

(2) For the conjugate poles on the imaginary axis

$$
\frac{\omega_{n}^{2}}{s^{2}+\omega_{n}^{2}} \Longrightarrow \frac{\omega_{n}^{2}}{s^{2}+2 \varepsilon \omega_{n} s+\omega_{n}^{2}} .
$$

$\varepsilon$ is a positive constant sufficiently small.

\section{Experimental Verification}

In this section, attitude tracking of a quadrotor aircraft is implemented to verify the effectiveness of the proposed control strategy. The modified Rodrigues parameters (MRPs) are applied to represent the attitude [33]. The attitude tracking error model is described as follows:

$$
\begin{aligned}
\dot{\tilde{\sigma}}= & G(\widetilde{\sigma}) \widetilde{\omega} \\
\dot{\tilde{\omega}}= & J^{-1}\left[-\left(\widetilde{\omega}+\widetilde{R} \omega_{\mathrm{d}}\right) J\left(\widetilde{\omega}+\widetilde{R} \omega_{\mathrm{d}}\right)+F u\right] \\
& -\left(\widetilde{R} \dot{\omega}_{\mathrm{d}}-[\widetilde{\omega} \times] \widetilde{R} \omega_{\mathrm{d}}\right),
\end{aligned}
$$

with the MRPs and angular velocity error defined as

$$
\begin{aligned}
& \tilde{\sigma}=\sigma \oplus \sigma_{\mathrm{d}}^{-1}, \\
& \widetilde{\omega}=\omega-\widetilde{R} \omega_{\mathrm{d}},
\end{aligned}
$$


TABLE 1: Parameters of the quadrotor aircraft.

\begin{tabular}{|c|c|c|c|c|}
\hline Parameter & Definition & Value & Error & Unit \\
\hline$\overline{C_{T}}$ & Coefficients of thrust & 0.012 & \pm 0.003 & \\
\hline$C_{Q}$ & Coefficients of torque & $0.93 \times 10^{-3}$ & $\pm 0.2 \times 10^{-3}$ & \\
\hline$\rho$ & Density of air & 1.184 & & $\mathrm{Kg} \cdot \mathrm{m}^{-3}$ \\
\hline$A$ & Propeller's disc area & 0.0515 & \pm 0.002 & $\mathrm{~m}^{2}$ \\
\hline$r$ & Propeller's radius & 0.128 & \pm 0.001 & $\mathrm{~m}$ \\
\hline$l$ & Rotor displacement from the center & 0.25 & \pm 0.01 & $\mathrm{~m}$ \\
\hline$J_{\phi}$ & Rotational inertia of roll axis & 0.014 & \pm 0.002 & $\mathrm{Kg} \cdot \mathrm{m}^{2}$ \\
\hline$J_{\theta}$ & Rotational inertia of pitch axis & 0.014 & \pm 0.002 & $\mathrm{Kg} \cdot \mathrm{m}^{2}$ \\
\hline$J_{\psi}$ & Rotational inertia of yaw axis & 0.024 & \pm 0.004 & $\mathrm{Kg} \cdot \mathrm{m}^{2}$ \\
\hline$\omega_{T}$ & Basic rotational speed of the rotor & 215 & \pm 5 & $\mathrm{rad} / \mathrm{s}$ \\
\hline
\end{tabular}

where $\sigma, \omega$, and $J$ are MRPs, angular velocity, and the inertia matrix, respectively. $G(\sigma)$ is a nonsingular matrix defined in [33]. $\sigma_{\mathrm{d}}^{-1}$ is known as inverse of $\sigma_{\mathrm{d}}$, which is extracted as $\sigma_{\mathrm{d}}^{-1}=-\sigma_{\mathrm{d}}$, and $\widetilde{R}=R R_{\mathrm{d}}^{\mathrm{T}}$ is known as the error of attitude transition matrix. The operator $\oplus$ denotes the production of MRPs. The control input is defined as $u=$ $\left[\begin{array}{lll}\omega_{\phi} & \omega_{\theta} & \omega_{\psi}\end{array}\right]^{\mathrm{T}}$. Then, the rotational speeds of each propeller are

$$
\begin{aligned}
& \omega_{1}=\omega_{T}+\omega_{\theta}+\omega_{\psi}, \\
& \omega_{2}=\omega_{T}+\omega_{\phi}-\omega_{\psi}, \\
& \omega_{3}=\omega_{T}-\omega_{\theta}+\omega_{\psi}, \\
& \omega_{4}=\omega_{T}-\omega_{\phi}-\omega_{\psi},
\end{aligned}
$$

and by assuming that the value of $u$ is smaller than that of $\omega_{T}$, we finally get the matrix $F$ as

$$
F=\operatorname{diag}\left(4 C_{T} \rho A r^{2} l \omega_{T}, 4 C_{T} \rho A r^{2} l \omega_{T}, 8 C_{\mathrm{Q}} \rho A r^{3} \omega_{T}\right) .
$$

The related parameter descriptions are shown in Table 1 [34].

4.1. Control System Design and Implementation. Assume that the nominal inertia is $J_{0}$ and inertia error as $\Delta J=J-J_{0}$. Meanwhile, the nominal value of $F$ is given as $F_{0}$, and its error is defined as $\Delta F=F-F_{0}$. Then, we can use the feedback linearization

$$
\begin{aligned}
u= & v+F_{0}^{-1} L\left(\widetilde{\omega}+\widetilde{R} \omega_{\mathrm{d}}\right) \operatorname{vec}\left(J_{0}\right) \\
& +F_{0}^{-1} J_{0}\left(\widetilde{R} \dot{\omega}_{\mathrm{d}}-[\widetilde{\omega} \times] \widetilde{R} \omega_{\mathrm{d}}\right),
\end{aligned}
$$

to reduce the system dynamics to

$$
F_{0}^{-1} J_{0} \dot{\tilde{\omega}}=v+d+f
$$

where the definitions of the operators $L(\cdot)$ and vec $(\cdot)$ satisfy $L\left(\widetilde{\omega}+\widetilde{R} \omega_{\mathrm{d}}\right) \operatorname{vec}\left(J_{0}\right)=\left(\widetilde{\omega}+\widetilde{R} \omega_{\mathrm{d}}\right) \times J_{0}\left(\widetilde{\omega}+\widetilde{R} \omega_{\mathrm{d}}\right)$ and operator vec(.) is a vector that contains all the components of the symmetric square matrix. The external disturbance $d$ satisfies $\|d\| \leq \bar{d}$. The internal uncertainty is defined as

$$
\begin{aligned}
& f \\
& =-\left[\delta \dot{\tilde{\omega}}+L\left(\widetilde{\omega}+\widetilde{R} \omega_{\mathrm{d}}\right) \delta^{*}+\delta\left(\widetilde{R} \dot{\omega}_{\mathrm{d}}-[\widetilde{\omega} \times] \widetilde{R} \omega_{\mathrm{d}}\right)\right],
\end{aligned}
$$

where $\delta \triangleq\left(F F_{0}\right)^{-1}\left(F_{0} \Delta J-\Delta F J_{0}\right)$.

By substituting (53) into (54), we have

$$
\begin{aligned}
f & =\left(I_{3}+\delta J_{0}^{-1} F_{0}\right)^{-1}\left[-\delta J_{0}^{-1} F_{0}(v+d)\right. \\
& \left.-L\left(\widetilde{\omega}+\widetilde{R} \omega_{\mathrm{d}}\right) \delta^{*}-\delta\left(\widetilde{R} \dot{\omega}_{\mathrm{d}}-[\widetilde{\omega} \times] \widetilde{R} \omega_{\mathrm{d}}\right)\right] .
\end{aligned}
$$

Since $\|\widetilde{R}\|, \omega_{\mathrm{d}}$, and $\dot{\omega}_{\mathrm{d}}$ are all bounded and control input $v$ can be rewritten into the form of state feedback, the internal uncertainty $f$ satisfies Assumption 3 .

According to linearized model (53), the observer can be designed as

$$
\widehat{d}=-Q(s) v+s Q(s) F_{0}^{-1} J_{0} \widetilde{\omega},
$$

and the backstepping controller is designed as

$$
\begin{aligned}
u= & -\left(1+k_{1} k_{2}\right) \widetilde{\sigma}-\left(k_{2}+F_{0}^{-1} J_{0} k_{1} G(\widetilde{\sigma})\right) \widetilde{\omega}-\widehat{d} \\
& +F_{0}^{-1} L\left(\widetilde{\omega}+\widetilde{R} \omega_{\mathrm{d}}\right) J_{0}^{*} \\
& +F_{0}^{-1} J_{0}\left(\widetilde{R} \dot{\omega}_{\mathrm{d}}-[\widetilde{\omega} \times] \widetilde{R} \omega_{\mathrm{d}}\right) .
\end{aligned}
$$

For the variable $\widetilde{\Omega}=\widetilde{\omega}+k_{1} \widetilde{\sigma}$ and the Lyapunov function defined as $V_{\text {rot }}=2 \ln \left(1+\widetilde{\sigma}^{\mathrm{T}} \widetilde{\sigma}\right)+(1 / 2) \widetilde{\Omega}^{\mathrm{T}}\left(F_{0}^{-1} J_{0}\right) \widetilde{\Omega}$, we have

$$
\dot{V}_{2} \leq-\lambda_{\text {min }}\left(k_{1}\right)\|\widetilde{\sigma}\|^{2}-\lambda_{\text {min }}\left(k_{2}\right)\|\widetilde{\Omega}\|^{2}+\|\widetilde{\Omega}\|\|\widetilde{\mathrm{d}}\| .
$$

For the controller in (57), the parameters are selected as $k_{1}=1.5$ and $k_{2}=9.0$; the system dynamics and expression of uncertainty are given as follows:

$$
\begin{aligned}
& {\left[F_{0}^{-1} J_{0} s+\left(k_{2}+F_{0}^{-1} J_{0} k_{1} G(\widetilde{\sigma})\right)+\left(1+k_{1} k_{2}\right) G(\widetilde{\sigma}) \frac{1}{s}\right]} \\
& \cdot \widetilde{\omega}=d+f-\widehat{d}, \\
& f=-\left[\delta \dot{\widetilde{\omega}}-L\left(\widetilde{\omega}+\widetilde{R} \omega_{\mathrm{d}}\right) \delta^{*}-\delta\left(\widetilde{R} \dot{\omega}_{\mathrm{d}}-[\widetilde{\omega} \times] \widetilde{R} \omega_{\mathrm{d}}\right)\right] .
\end{aligned}
$$




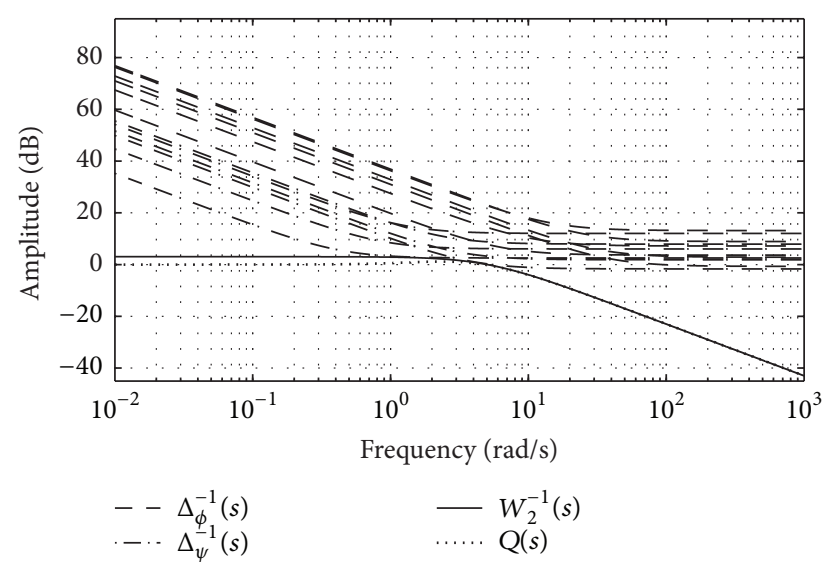

FIGURE 3: System uncertainties and weighting function constraint.

From the analysis in Section 3.3, we get the nominal model of equivalent system as

$$
P_{\Delta n}=\frac{4 s}{4 F_{0}^{-1} J_{0} s^{2}+\left(4 k_{2}+F_{0}^{-1} J_{0} k_{1}\right) s+\left(1+k_{1} k_{2}\right)},
$$

while the equivalent system is shown as

$$
\begin{aligned}
& P_{\Delta}(s) \\
& =\frac{4 s}{\left(4 F_{0}^{-1} J_{0}+\delta\right) s^{2}+\left(4 k_{2}+F_{0}^{-1} J_{0} k_{1}+4 k_{3}\right) s+\left(1+k_{1} k_{2}\right)},
\end{aligned}
$$

where $k_{3}=-\left(\partial L\left(\widetilde{\omega}+\widetilde{R} \omega_{\mathrm{d}}\right) \delta^{*}+\delta\left(\widetilde{R} \dot{\omega}_{\mathrm{d}}-[\widetilde{\omega} \times] \widetilde{R} \omega_{\mathrm{d}}\right)\right) / \partial \widetilde{\omega}$.

Consider that the structure of quadrotor is axially symmetric; the corresponding parameters of pitch and roll axes are the same. Thus, weighting function $W_{2}(s)$ can be determined by pitch (roll) axis and yaw axis. The selection of $W_{2}(s)$ should contain the system uncertainties with all the parameters perturbation. It is also required that the designed $Q$ filter has at least $-30 \mathrm{~dB}$ attenuation against measurement noise of gyroscope larger than $42 \mathrm{~Hz}$. Figure 3 shows the frequency response of $\Delta(s)$ according to the parameters perturbation. It is illustrated that, for all the possible parameters, the weighting function satisfies $W_{2}^{-1}(s) \leq \Delta_{\phi}(s), W_{2}^{-1}(s) \leq$ $\Delta_{\psi}(s)$. Then, the optimized $Q$ filter is obtained while $\gamma=8.1$ :

$$
Q(s)=\frac{7.1 s+11.415}{s^{2}+7.1 s+11.415} .
$$

4.2. Simulations. Numerical simulations are presented in MATLAB/Simulink to illustrate the efficacy of the proposed strategy. The simulation period is $5 \mathrm{~ms}$, the same as that in experiments. We consider the parameters and their uncertainties depicted in Table 1 . The desired MRPs are given as

$$
\begin{aligned}
\sigma_{\mathrm{d} 1} & =0.1 \sin \left(\frac{\pi t}{15}+\frac{\pi}{2}\right), \\
\sigma_{\mathrm{d} 2} & =0.1 \sin \left(\frac{\pi t}{15}-\frac{\pi}{2}\right), \\
\sigma_{\mathrm{d} 3} & =0.1 \sin \left(\frac{\pi t}{15}\right) ;
\end{aligned}
$$

hence, from the kinematics of MRPs, we get

$$
\begin{aligned}
& \omega_{\mathrm{d}}=G^{-1}\left(\sigma_{\mathrm{d}}\right) \dot{\sigma}_{\mathrm{d}}, \\
& \dot{\omega}_{\mathrm{d}}=G^{-1}\left(\sigma_{\mathrm{d}}\right)\left[\ddot{\sigma}_{\mathrm{d}}-\bar{G}\left(\sigma_{\mathrm{d}}, \dot{\sigma}_{\mathrm{d}}\right) \omega_{\mathrm{d}}\right],
\end{aligned}
$$

where $\bar{G}\left(\sigma_{\mathrm{d}}, \dot{\sigma}_{\mathrm{d}}\right)$ is the time-derivative of $G\left(\sigma_{\mathrm{d}}\right)$.

The external disturbances on the dynamics are as follows:

$$
\begin{aligned}
& d_{1}=0.1 \sin \left(\frac{\pi t}{2}\right)+0.1 \sin \left(\frac{\pi t}{10}\right)+0.3, \\
& d_{2}=0.1 \sin \left(\frac{\pi t}{2}\right)+0.1 \cos \left(\frac{\pi t}{10}\right)+0.4, \\
& d_{3}=0.1 \sin \left(\frac{\pi t}{2}\right)+0.1 \cos \left(\frac{\pi t}{10}+\frac{\pi}{4}\right)+0.5,
\end{aligned}
$$

which contains constant and sine components with both low and high frequencies.

The measurement noise is taken into account in this simulation. Here, we add the practical noise from the sensors to the feedback channel. The initial condition is $\sigma\left(t_{0}\right)=$ $\left[\begin{array}{lll}0.1 & 0.15 & 0.05\end{array}\right]^{\mathrm{T}}, \omega\left(t_{0}\right)=\left[\begin{array}{lll}0 & 0 & 0\end{array}\right]^{\mathrm{T}}$. Note that the controller parameters are $k_{1}=10$ and $k_{2}=0.5$. Meanwhile, a nonlinear feedback controller in (57) without $\widehat{d}$ and a traditional DOB with first-order $Q$ filter are also carried out in this simulation to compare with the proposed strategy. The bandwidth of traditional DOB is 15 , which is selected to be as large as possible to guarantee both disturbance rejection performance and robustness against measurement noise.

Figure 4 shows the tracking effect. It is illustrated that the nonlinear feedback controller without DOB cannot suppress the influence caused by internal uncertainties and external disturbances. The compound disturbances acting on system dynamics will cause an obvious tracking error. The approximation of compound disturbances in Figure 5 illustrates that the proposed DOB can estimate the compound disturbances successfully with noise of high frequency. Hence, with the compensation of the estimating disturbances, the proposed control strategy can enable the quadrotor to track the desired MRPs with better performance in Figure 4. Comparing with the proposed DOB, a traditional DOB is presented and the tracking errors of these two methods are shown in Figure 6. With the high frequency measurement noise, the bandwidth of traditional DOB cannot be selected to be larger than 15 , since the high gain will enlarge the influence caused by measurement noise and diverge the control system. However, the proposed DOB has stronger suppression ability against disturbances with low frequency, as well as attenuation against noise with high frequency. Consequently, the tracking performance with the proposed DOB is better than that with traditional DOB. The control performances of these methods are given specifically in Table 2 . The control structure with a DOB has higher tracking accuracy. The disturbances estimating error of the proposed robust DOB is less than that of traditional DOB. 

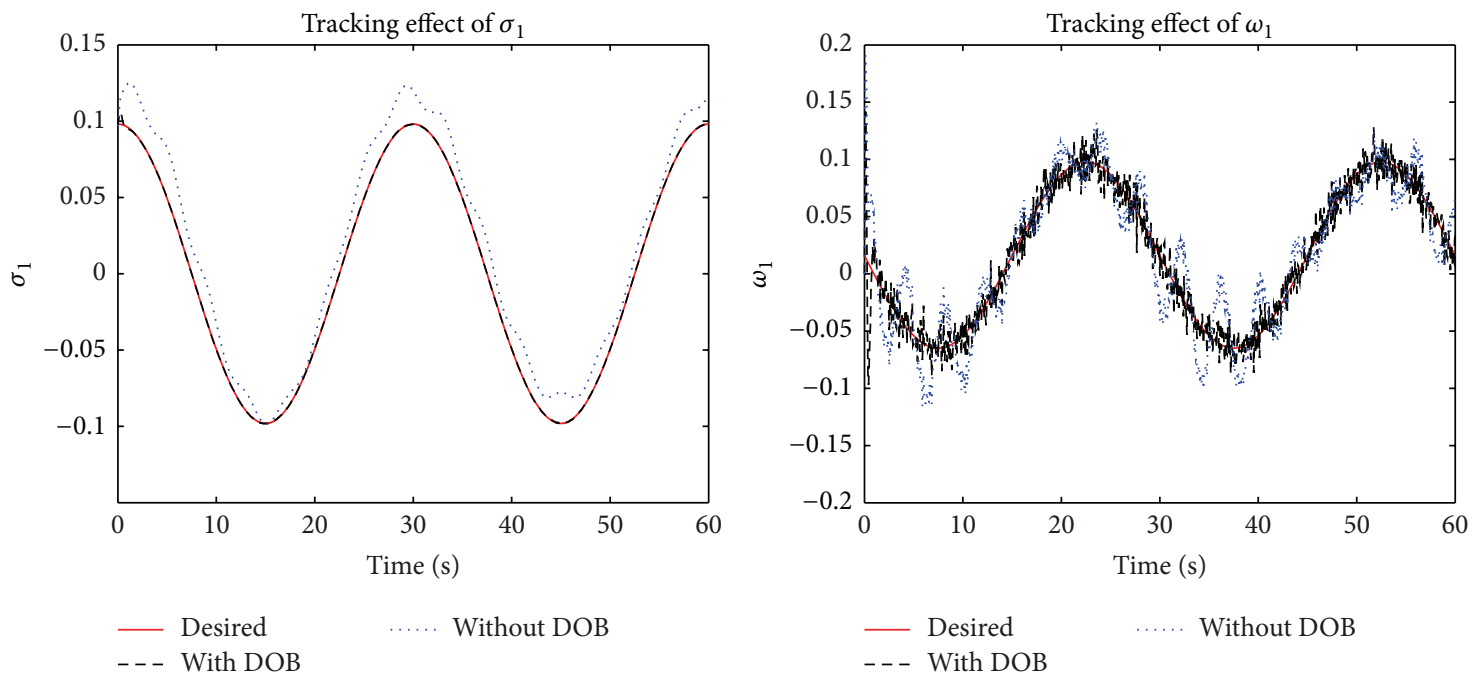

(a)
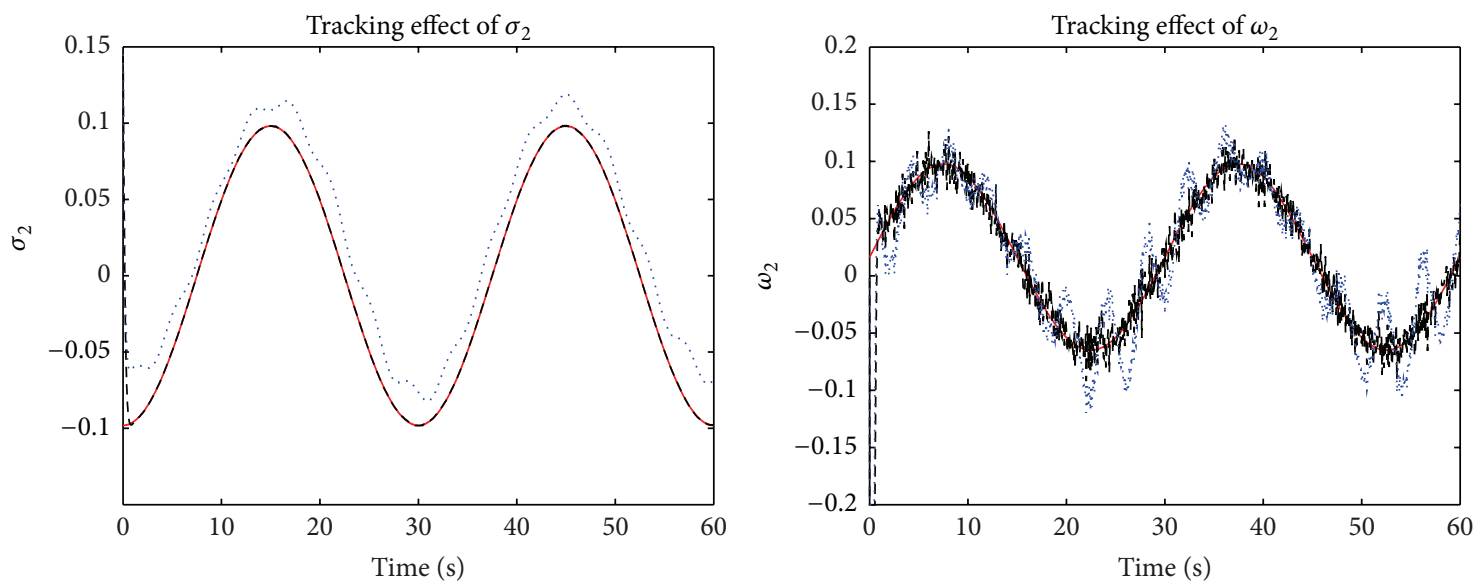

— Desired

Without DOB

- - - With DOB

$$
\text { - Desired }
$$

Without DOB

- - - With DOB

(b)
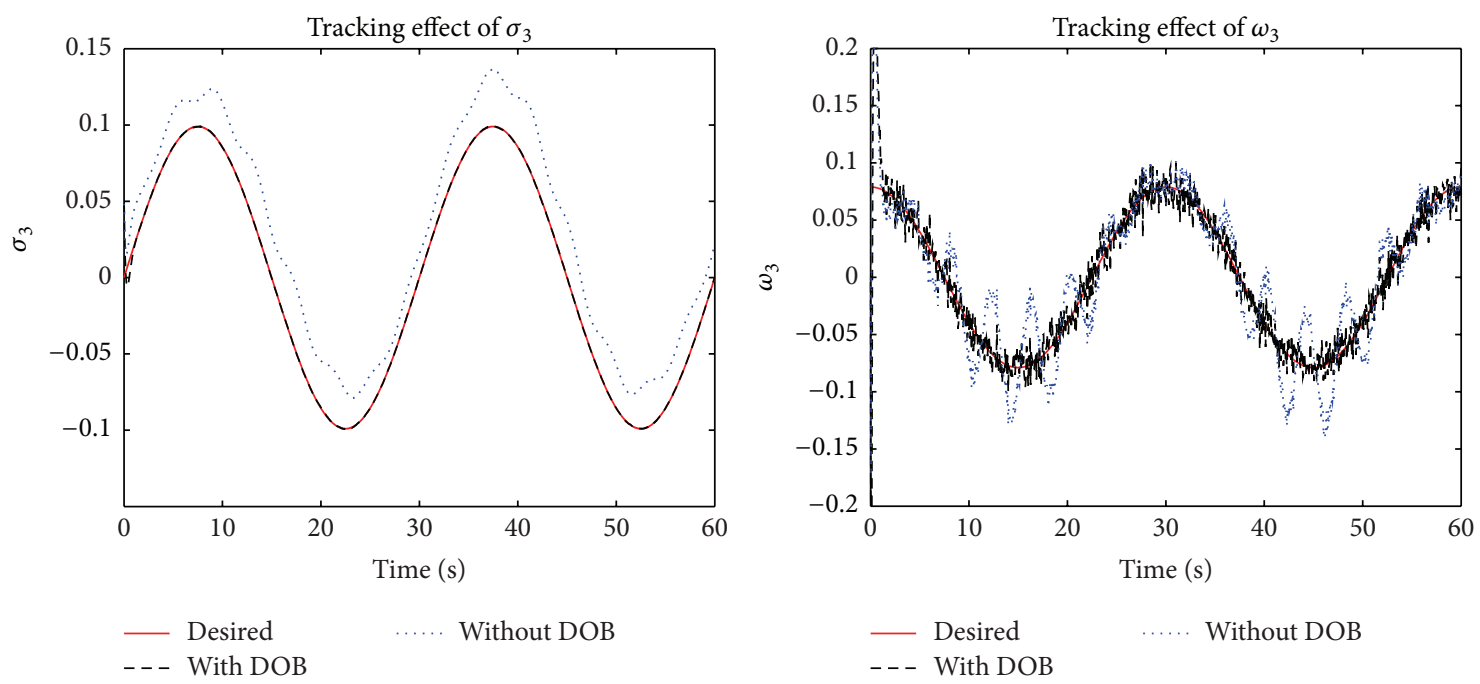

(c)

FIGURE 4: Tracking effects of desired attitude with and without DOB. 


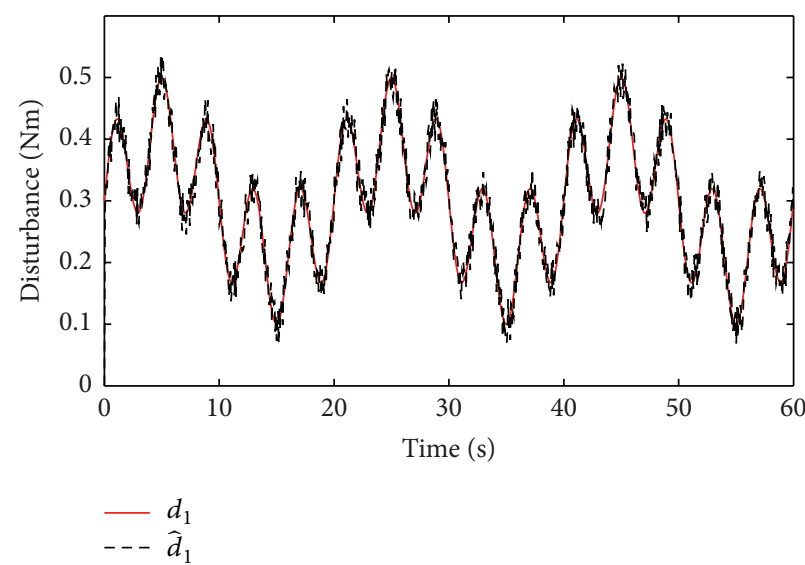

(a) Approximation effect of $d_{1}$

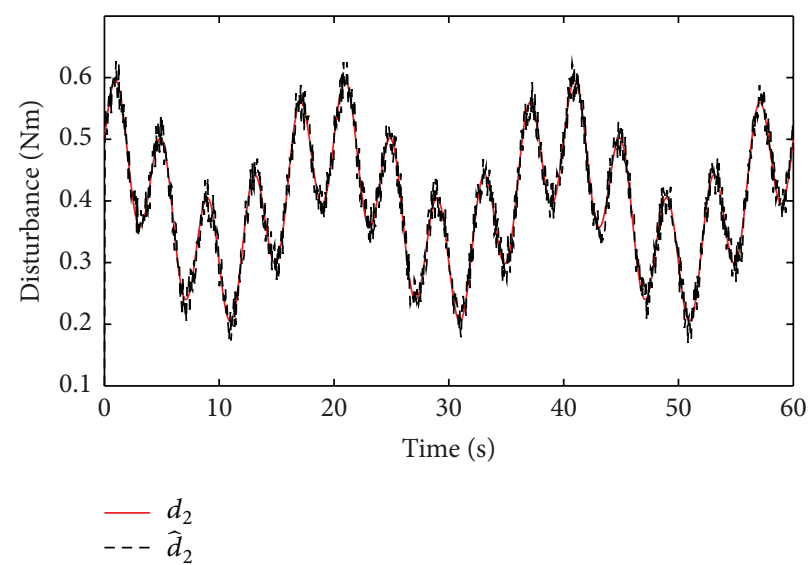

(b) Approximation effect of $d_{2}$

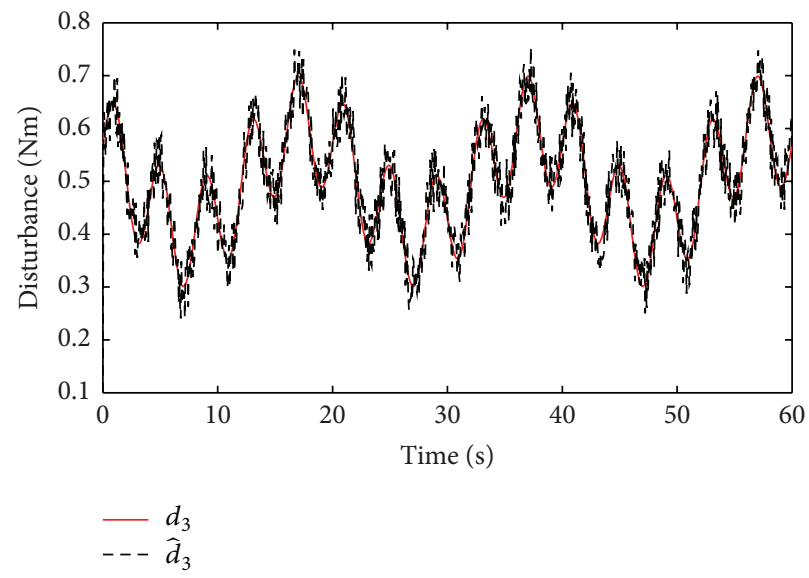

(c) Approximation effect of $d_{3}$

FIGURE 5: Approximation effect of disturbances.

TABLE 2: Comparison of control performances in simulations (RMS error).

\begin{tabular}{lccc}
\hline & $\widetilde{\sigma}$ & $\widetilde{\omega}$ & $\widetilde{\mathrm{d}}$ \\
\hline Without DOB & 0.04 & 0.05 & $\mathrm{Null}$ \\
Traditional DOB & $1.85 \times 10^{-2}$ & $0.12 \mathrm{rad} / \mathrm{s}$ & $0.17 \mathrm{~N} \cdot \mathrm{m}$ \\
Proposed DOB & $1.24 \times 10^{-2}$ & $0.07 \mathrm{rad} / \mathrm{s}$ & $0.13 \mathrm{~N} \cdot \mathrm{m}$ \\
\hline
\end{tabular}

4.3. Experimental Results. In the experiment, the desired attitude is expressed as follows:

$$
\begin{aligned}
\sigma_{\mathrm{d} 1} & =-0.03 \sin \left(\frac{\pi}{5} t\right), \\
\sigma_{\mathrm{d} 2} & =0.03 \cos \left(\frac{\pi}{5} t\right),
\end{aligned}
$$

and $\sigma_{\mathrm{d} 3}$ retains $0 . \omega_{\mathrm{d}}$ and $\dot{\omega}_{\mathrm{d}}$ are acquired by (64).

It can be illustrated in (53) and (54) that even if there are no external disturbances, the existing internal uncertainties will also bring the system with an equivalent disturbance $f$. In Figure 7, we find that, with the action of DOB, the
TABLE 3: Comparison of control performances (RMS error).

\begin{tabular}{lccc}
\hline & $\sigma_{1}$ & $\sigma_{2}$ & $\sigma_{3}$ \\
\hline Traditional DOB & $1.4 \times 10^{-3}$ & $1.6 \times 10^{-3}$ & $2.2 \times 10^{-3}$ \\
Proposed DOB & $7.9 \times 10^{-4}$ & $7.1 \times 10^{-4}$ & $2.9 \times 10^{-4}$ \\
\hline
\end{tabular}

tracking error caused by internal uncertainties can be suppressed successfully. At time of 75 and 90 seconds, external disturbances are exerted on the quadrotor. The proposed $\mathrm{DOB}$ can estimate and eliminate the disturbance quickly and accurately. Also, from the enlarged view of Figure 7, we find the convergence speed of the DOB is less than 2 seconds. The attitude error is expressed in Figure 8 and the comparison of the attitude tracking performances is shown in Table 3 . The attitude errors are expressed in Figure 8 and the comparison of the attitude tracking performances is shown in Table 3. The control accuracy is much higher with the proposed DOB. Since there exists property of coupling among the 3 axes of the attitude, when we exert external disturbances on the axes of pitch or roll, it will in turn affect the other two axes. However, the proposed $\mathrm{DOB}$ can also suppress the influence caused by coupling property. Experimental results validate that the 


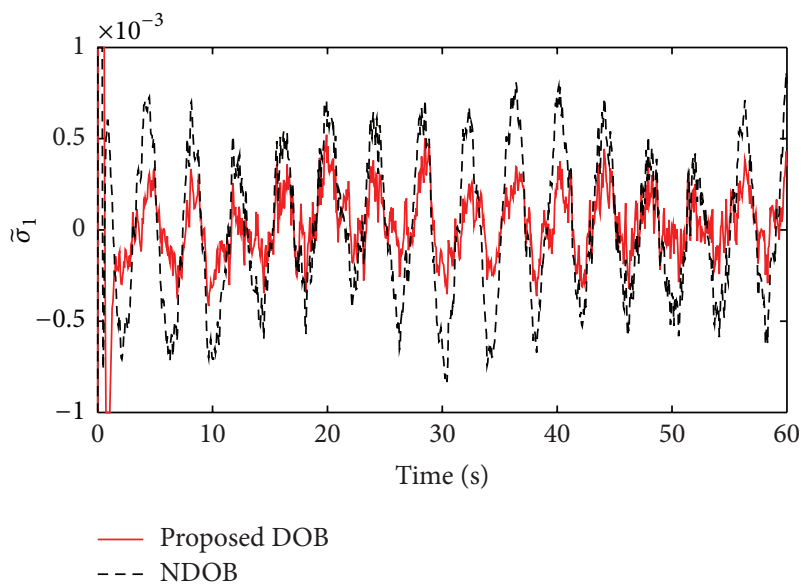

(a) Tracking error of $\sigma_{1}$

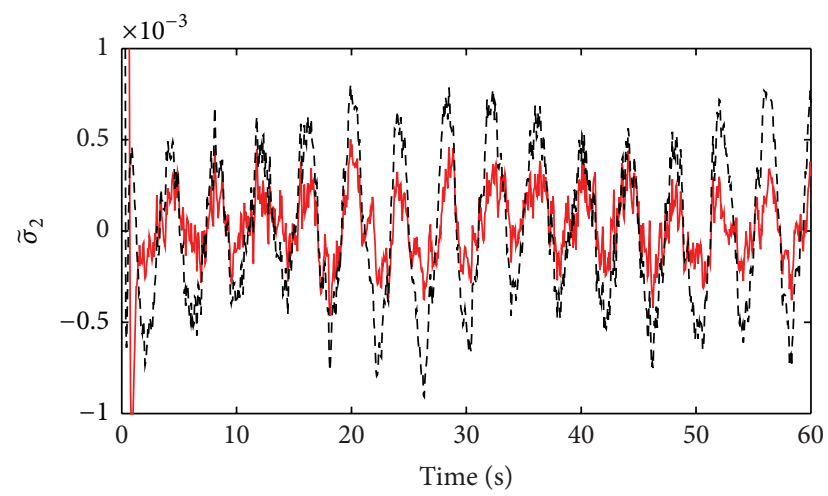

— Proposed DOB

- - - NDOB

(b) Tracking error of $\sigma_{2}$

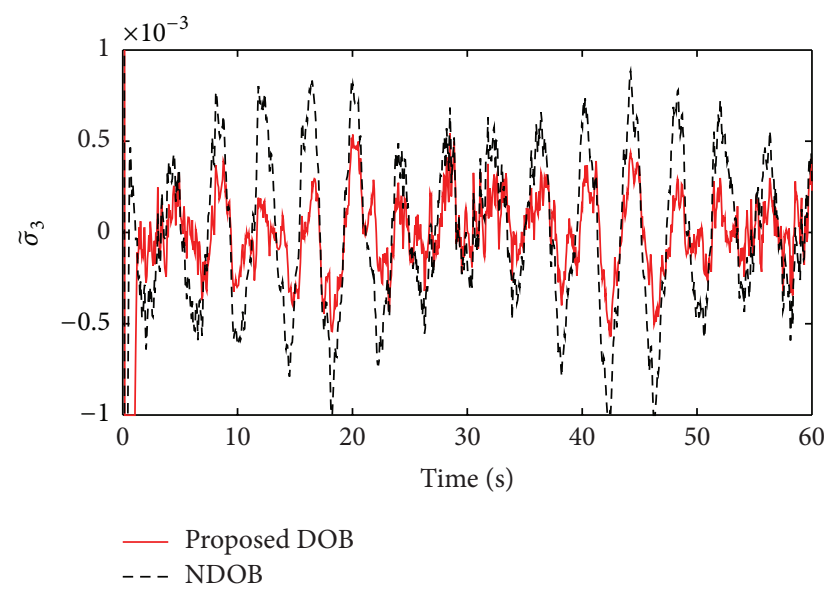

(c) Tracking error of $\sigma_{3}$

FIGURE 6: Tracking error comparison of the proposed DOB and traditional DOB.

proposed control strategy can obtain strong disturbance rejection performance against external disturbances as well as good tracking performance. The robust stability of the closedloop system can be guaranteed based on the proposed DOB optimization strategy.

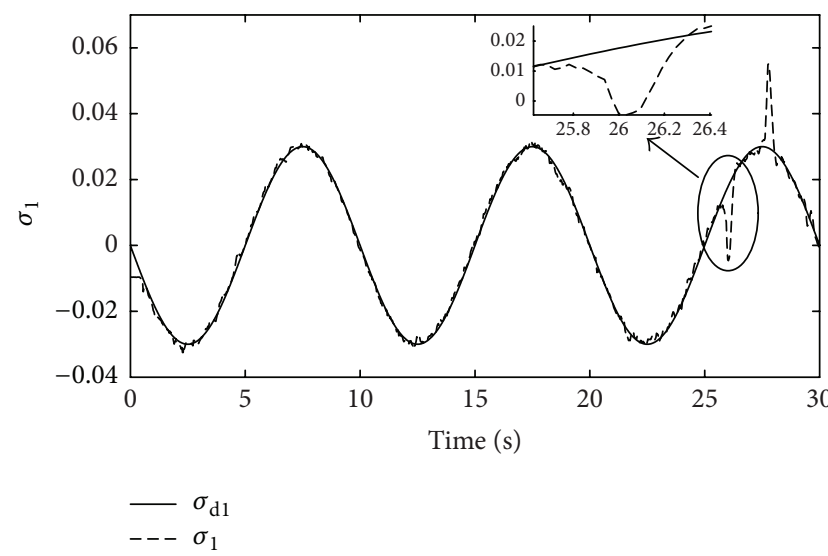

(a) Tracking effect of $\sigma_{1}$

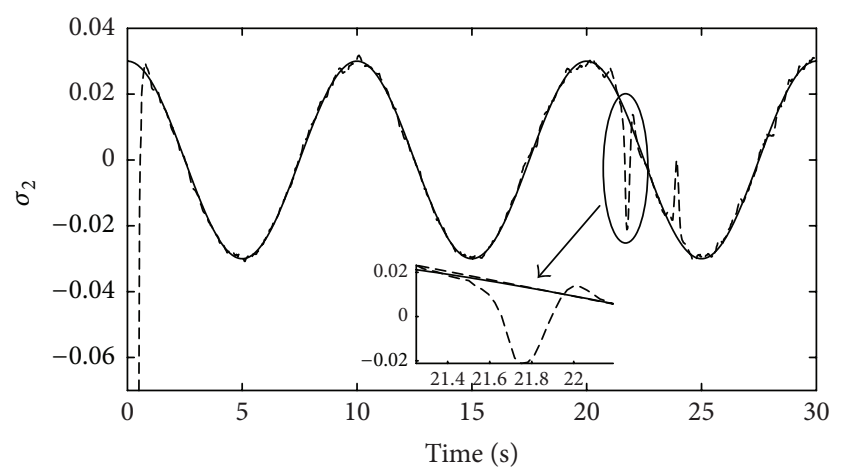

$-\sigma_{\mathrm{d} 2}$

(b) Tracking effect of $\sigma_{2}$

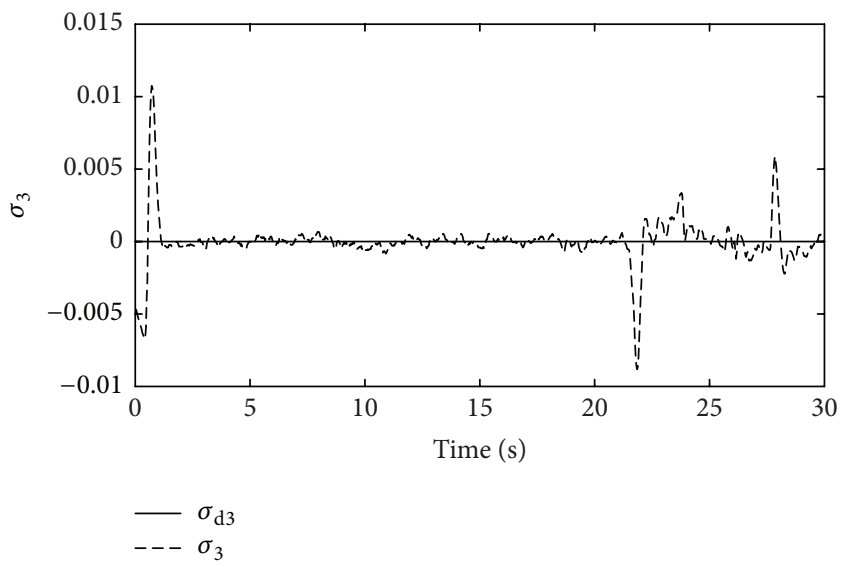

(c) Tracking effect of $\sigma_{3}$

FIgURE 7: Tracking effect of MRPs with DOB.

\section{Conclusions}

This paper proposes a disturbance rejection control strategy for nonlinear systems with robust DOB. First, a DOB with partial feedback linearization and a low-pass filter is proposed for nonlinear dynamic model under relaxed restrictions of the generalized disturbance. Then, the outerloop backstepping controller is designed for desired tracking 


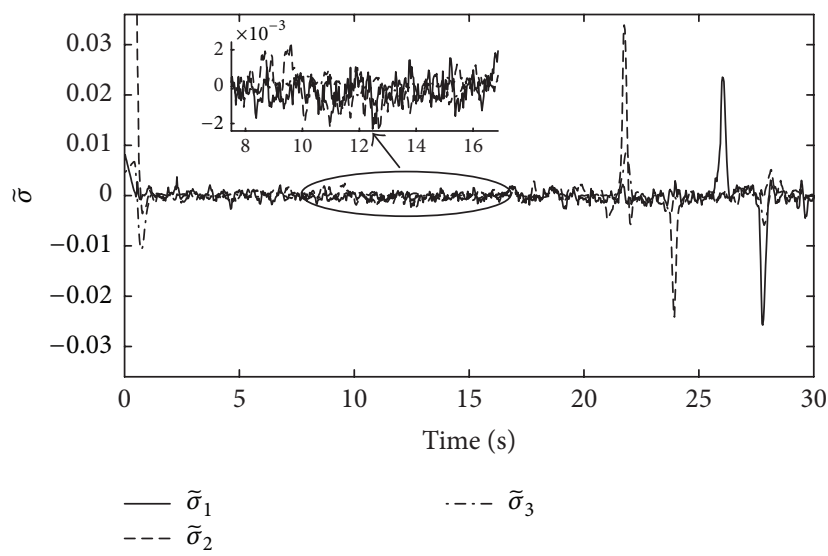

FIGURE 8: Tracking error of the proposed method.

performance. By analyzing the influence of outer controller on the inner-loop observer parameter, the robust stability constraint condition is proposed to guarantee the robust stability of the closed-loop system. Experimental results on an aircraft show that the proposed strategy can increase the control accuracy effectively. The optimized DOB can eliminate the external disturbances effectively to increase the control accuracy. Meanwhile, the proposed parameters optimization strategy can guarantee the robust stability of the closed-loop system.

\section{Competing Interests}

The authors declare that there are no competing interests regarding the publication of this paper.

\section{References}

[1] Z. Gao, "On the centrality of disturbance rejection in automatic control," ISA Transactions, vol. 53, no. 4, pp. 850-857, 2014.

[2] M. L. Corradini, V. Fossi, A. Giantomassi, G. Ippoliti, S. Longhi, and G. Orlando, "Discrete time sliding mode control of robotic manipulators: development and experimental validation," Control Engineering Practice, vol. 20, no. 8, pp. 816-822, 2012.

[3] J. Yang, J. Su, S. Li, and X. Yu, "High-order mismatched disturbance compensation for motion control systems via a continuous dynamic sliding-mode approach," IEEE Transactions on Industrial Informatics, vol. 10, no. 1, pp. 604-614, 2014.

[4] L.-B. Li, L.-L. Sun, S.-Z. Zhang, and Q.-Q. Yang, "Speed tracking and synchronization of multiple motors using ring coupling control and adaptive sliding mode control," ISA Transactions, vol. 58, pp. 635-649, 2015.

[5] K. J. Åström and B. Wittenmark, Adaptive Control, Courier Corporation, 2013.

[6] Z. Li, S. Deng, C.-Y. Su et al., "Decentralised adaptive control of cooperating Robotic manipulators with disturbance observers," IET Control Theory and Applications, vol. 8, no. 7, pp. 515-521, 2014.

[7] F. Motallebzadeh, S. Ozgoli, and H. R. Momeni, "Multilevel adaptive control of nonlinear interconnected systems," ISA Transactions, vol. 54, pp. 83-91, 2015.
[8] K. Zhou, J. C. Doyle, and K. Glover, Robust and Optimal Control, vol. 40, Prentice Hall, Upper Saddle River, NJ, USA, 1996.

[9] L. Lu and B. Yao, "Energy-saving adaptive robust control of a hydraulic manipulator using five cartridge valves with an accumulator," IEEE Transactions on Industrial Electronics, vol. 61, no. 12, pp. 7046-7054, 2014.

[10] J. P. Kolhe, M. Shaheed, T. S. Chandar, and S. E. Talole, "Robust control of robot manipulators based on uncertainty and disturbance estimation," International Journal of Robust and Nonlinear Control, vol. 23, no. 1, pp. 104-122, 2013.

[11] R.-J. Wai and R. Muthusamy, "Design of fuzzy-neural-networkinherited backstepping control for robot manipulator including actuator dynamics," IEEE Transactions on Fuzzy Systems, vol. 22, no. 4, pp. 709-722, 2014.

[12] J. L. Meza, V. Santibáñez, R. Soto, and M. A. Llama, "Fuzzy selftuning PID semiglobal regulator for robot manipulators," IEEE Transactions on Industrial Electronics, vol. 59, no. 6, pp. 27092717, 2012.

[13] J. Addeh, A. Ebrahimzadeh, M. Azarbad, and V. Ranaee, "Statistical process control using optimized neural networks: a case study," ISA Transactions, vol. 53, no. 5, pp. 1489-1499, 2014.

[14] D. Xing, J. Su, Y. Liu, and J. Zhong, "Robust approach for humanoid joint control based on a disturbance observer," IET Control Theory \& Applications, vol. 5, no. 14, pp. 1630-1636, 2011.

[15] S. Lichiardopol, N. van de Wouw, and H. Nijmeijer, "Robust disturbance estimation for human-robotic comanipulation," International Journal of Robust and Nonlinear Control, vol. 24, no. 12, pp. 1772-1796, 2014.

[16] J. N. Yun and J.-B. Su, "Design of a disturbance observer for a two-link manipulator with flexible joints," IEEE Transactions on Control Systems Technology, vol. 22, no. 2, pp. 809-815, 2014.

[17] A. Mohammadi, M. Tavakoli, H. J. Marquez, and F. Hashemzadeh, "Nonlinear disturbance observer design for robotic manipulators," Control Engineering Practice, vol. 21, no. 3, pp. 253-267, 2013.

[18] Z. Ma and J. Su, "Robust uncalibrated visual servoing control based on disturbance observer," ISA Transactions, vol. 59, pp. 193-204, 2015.

[19] J. Yang, S. Li, C. Sun, and L. Guo, "Nonlinear-disturbanceobserver-based robust flight control for airbreathing hypersonic vehicles," IEEE Transactions on Aerospace and Electronic Systems, vol. 49, no. 2, pp. 1263-1275, 2013.

[20] L. Wang and J. Su, "Disturbance rejection control of a morphing UAV," in Proceedings of the American Control Conference (ACC '13), pp. 4307-4312, Washington, DC, USA, June 2013.

[21] K. Yang, Y. Choi, and W. K. Chung, "On the tracking performance improvement of optical disk drive servo systems using error-based disturbance observer," IEEE Transactions on Industrial Electronics, vol. 52, no. 1, pp. 270-279, 2005.

[22] H. Kim, H. Shim, and N. H. Jo, "Adaptive add-on output regulator for rejection of sinusoidal disturbances and application to optical disc drives," IEEE Transactions on Industrial Electronics, vol. 61, no. 10, pp. 5490-5499, 2014.

[23] C. Wang, X. Li, L. Guo, and Y. W. Li, "A nonlinear-disturbanceobserver-based DC-Bus voltage control for a hybrid AC/DC microgrid," IEEE Transactions on Power Electronics, vol. 29, no. 11, pp. 6162-6177, 2014.

[24] L. Wang and J. Su, "Disturbance rejection control for nonminimum phase systems with optimal disturbance observer," ISA Transactions, vol. 57, pp. 1-9, 2015. 
[25] W. Li and Y. Hori, "Vibration suppression using single neuronbased PI fuzzy controller and fractional-order disturbance observer," IEEE Transactions on Industrial Electronics, vol. 54, no. 1, pp. 117-126, 2007.

[26] S. Li, J. Li, and Y. Mo, "Piezoelectric multimode vibration control for stiffened plate using ADRC-based acceleration compensation," IEEE Transactions on Industrial Electronics, vol. 61, no. 12, pp. 6892-6902, 2014.

[27] K. Ohnishi, "A new servo method in mechatronics," Transactions of the Japan Society of Mechanical Engineers, vol. 107, pp. 83-86, 1987.

[28] Z.-J. Yang, S. Hara, S. Kanae, and K. Wada, "Robust output feedback control of a class of nonlinear systems using a disturbance observer," IEEE Transactions on Control Systems Technology, vol. 19, no. 2, pp. 256-268, 2011.

[29] W.-H. Chen, D. J. Ballance, P. J. Gawthrop, and J. O’Reilly, “A nonlinear disturbance observer for robotic manipulators," IEEE Transactions on Industrial Electronics, vol. 47, no. 4, pp. 932-938, 2000.

[30] W.-H. Chen, "Disturbance observer based control for nonlinear systems," IEEE/ASME Transactions on Mechatronics, vol. 9, no. 4, pp. 706-710, 2004.

[31] J. Han, "From PID to active disturbance rejection control," IEEE Transactions on Industrial Electronics, vol. 56, no. 3, pp. 900906, 2009.

[32] J. C. Doyle, K. Glover, P. P. Khargonekar et al., "State-space solutions to standard $h 2$ and $h 1$ control problems," IEEE Transactions on Automatic Control, vol. 34, no. 8, pp. 831-847, 1989.

[33] P. Tsiotras, "Further passivity results for the attitude control problem," IEEE Transactions on Automatic Control, vol. 43, no. 11, pp. 1597-1600, 1998.

[34] L. Wang and J. Su, "Switching control of attitude tracking on a quadrotor UAV for large-angle rotational maneuvers," in Proceedings of the IEEE International Conference on Robotics and Automation (ICRA '14), pp. 2907-2912, Hong Kong, June 2014. 


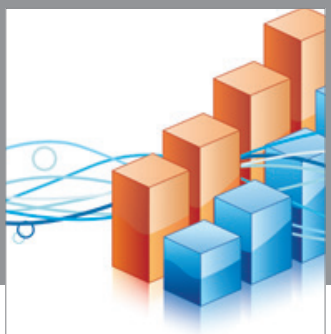

Advances in

Operations Research

vatem alat4

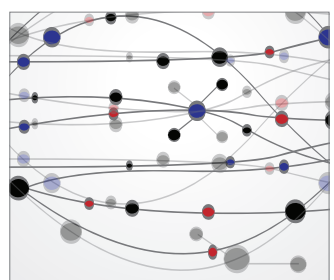

\section{The Scientific} World Journal
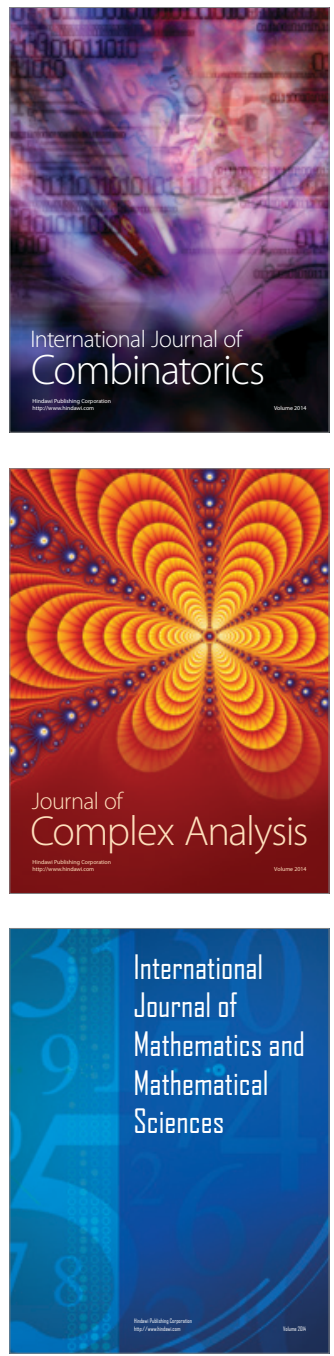
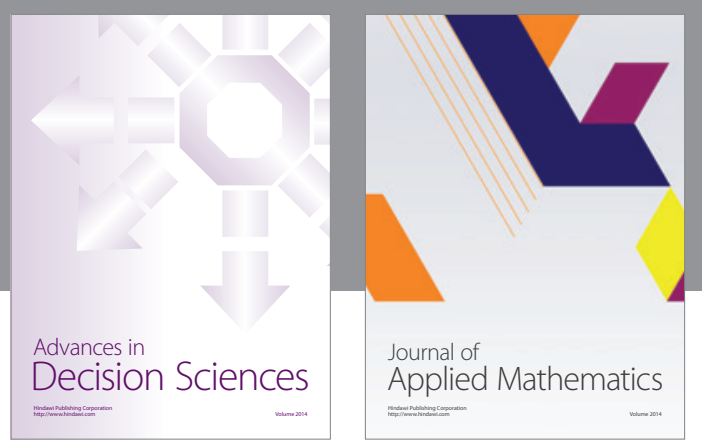

Algebra

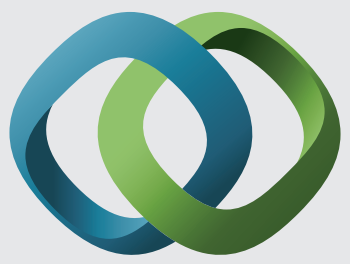

\section{Hindawi}

Submit your manuscripts at

http://www.hindawi.com
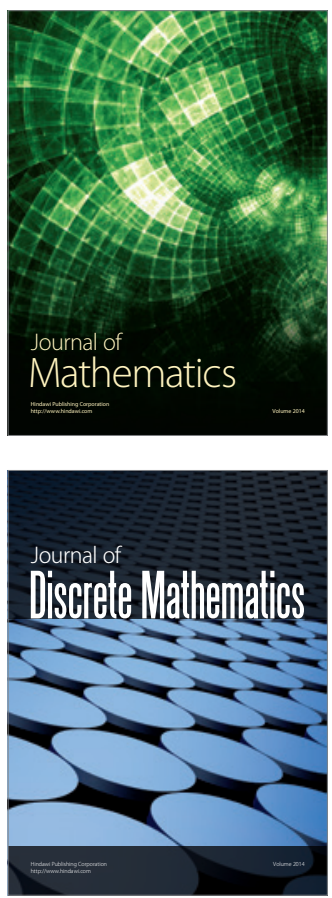

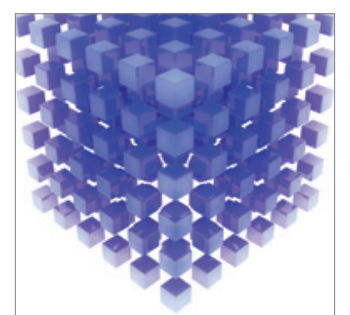

Mathematical Problems in Engineering
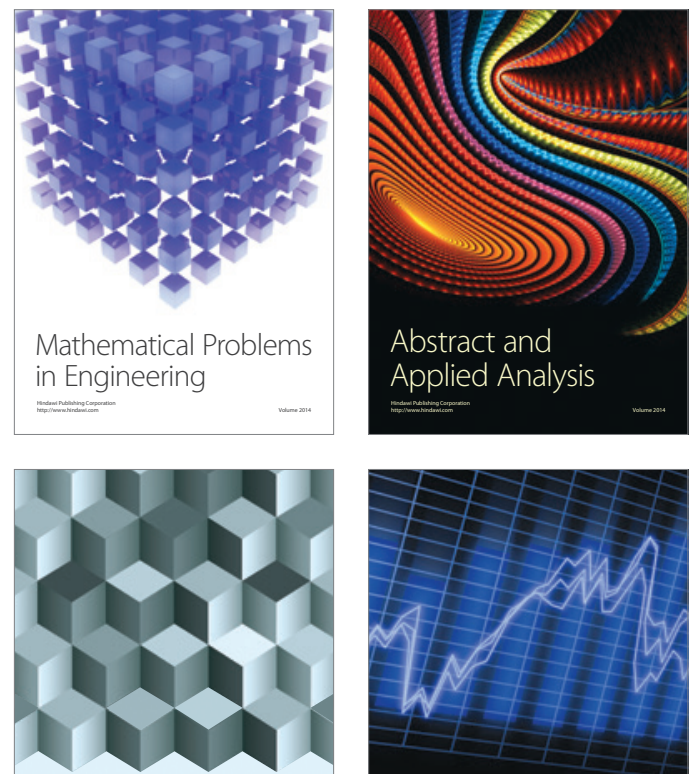

Journal of

Function Spaces

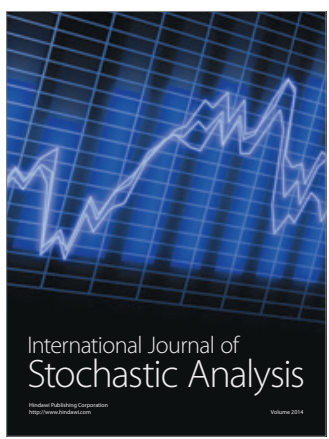

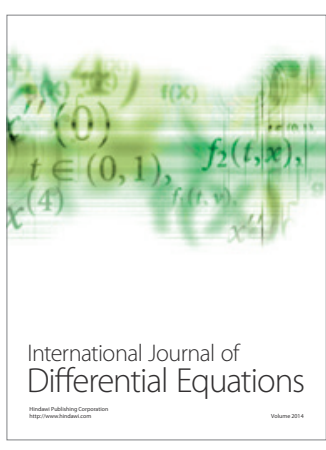
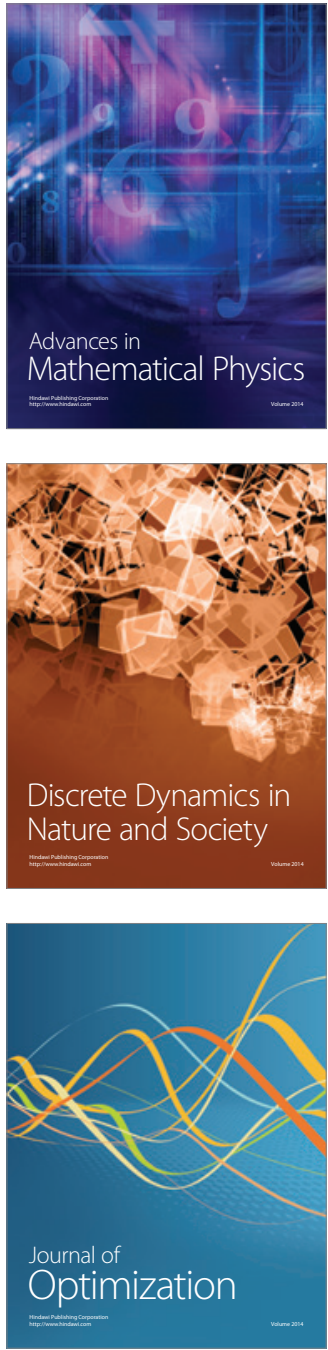